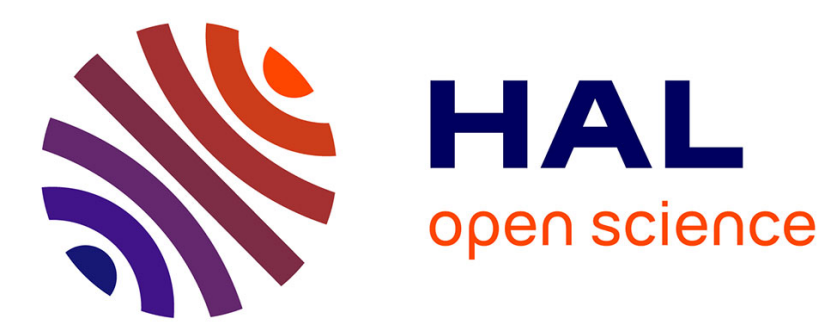

\title{
Préamplificateur refroidi rapide à faible bruit pour détecteur IR photoconducteur \\ P. Boissel
}

\section{To cite this version:}

P. Boissel. Préamplificateur refroidi rapide à faible bruit pour détecteur IR photoconducteur. Revue de Physique Appliquée, 1985, 20 (7), pp.523-526. 10.1051/rphysap:01985002007052300 . jpa-00245365

\section{HAL Id: jpa-00245365 https://hal.science/jpa-00245365}

Submitted on 1 Jan 1985

HAL is a multi-disciplinary open access archive for the deposit and dissemination of scientific research documents, whether they are published or not. The documents may come from teaching and research institutions in France or abroad, or from public or private research centers.
L'archive ouverte pluridisciplinaire HAL, est destinée au dépôt et à la diffusion de documents scientifiques de niveau recherche, publiés ou non, émanant des établissements d'enseignement et de recherche français ou étrangers, des laboratoires publics ou privés. 


\title{
Préamplificateur refroidi rapide à faible bruit pour détecteur IR photoconducteur
}

\author{
P. Boissel \\ Laboratoire de Photophysique Moléculaire(*), Bâtiment 213, Université de Paris-Sud, 91405 Orsay Cedex, France
}

(Reçu le 10 octobre 1984, révisé le 13 décembre 1984, accepté le 18 mars 1985)

\begin{abstract}
Résumé. - Nous avons réalisé un préamplificateur refroidi permettant d'obtenir un temps de réponse inférieur à $10 \mathrm{~ns}$ avec un détecteur infrarouge photoconducteur germanium cuivre, tout en conservant un rapport signal/bruit inférieur d'un facteur 3 seulement à celui d'un photoconducteur idéal.
\end{abstract}

\begin{abstract}
We have developed a cooled preamplifier for a photoconductive germanium copper infrared detector. We obtained a response time shorter than $10 \mathrm{~ns}$ with a signal to noise ratio lower only by a factor 3 than that of an ideal photoconductor.
\end{abstract}

La mise en œuvre d'un détecteur photoconducteur nécessite toujours un compromis entre temps de réponse et sensibilité. Pour diminuer la constante de temps, il faut diminuer la résistance de charge à cause des capacités parasites, mais cela entraîne une dégradation du rapport signal/bruit. En effet, l'amplitude du signal diminue comme $R_{\mathrm{c}}$ alors que le bruit NyquistJohnson ne diminue que comme $R_{\mathrm{c}}^{1 / 2}$. De plus, le bruit des amplificateurs de sortie devient prépondérant lorsque $R_{\mathrm{c}}$ devient trop faible.

Ce problème se pose particulièrement avec le détecteur germanium cuivre car il ne fonctionne qu'audessous de $20 \mathrm{~K}$. Les faibles puissances de refroidissement dont on dispose à ces températures imposent des fils de connection longs et fins pour diminuer les pertes thermiques ce qui entraîne une capacité parasite de ligne élevée.

La solution généralement adoptée [3] pour obtenir des temps de réponse de l'ordre de la nanoseconde est d'utiliser une ligne adaptée 50 ohms. La capacité parasite est alors uniquement celle du cristal détecteur qui est de l'ordre de $10 \mathrm{pF}$. Mais le rapport signal/bruit que l'on obtient (Tableau II) n'était pas suffisant pour les besoins de nos expériences $[1,2]$.

Nous avons donc été amenés à réaliser un préamplificateur-adaptateur d'impédance placé juste derrière le détecteur dans la partie froide du cryostat. Cette solution permet d'augmenter la charge du détecteur car il n'y a plus de capacité parasite de ligne. La sortie

(*) Laboratoire Associé à l'université de Paris-Sud. du cryostat se fait ensuite normalement sous faible impédance. La difficulté est que le préamplificateur doit fonctionner à des températures proches de $20 \mathrm{~K}$.

\section{Réalisation.}

L'élément détecteur est un cristal de germanium dopé au cuivre qualité « High Speed » de S.B.R.C. Son temps de réponse intrinsèque est de 0,3 à $0,8 \mathrm{~ns}$.

L'ensemble est refroidi par un réfrigérateur à hélium en cycle fermé "Air Products » permettant d'atteindre une température de 10 à $15 \mathrm{~K}$.

En ce qui concerne le préamplificateur, le premier problème a été de trouver les éléments àctifs fonctionnant à basse température. Seuls quelques transistors à effet de champ restent utilisables. On trouve dans la littérature un certain nombre d'études $[4,5]$ ou de réalisations [6, 7] de préamplificateurs refroidis, mais elles sont généralement orientées vers des buts différents : recherche du bruit de fond minimum (limite du détecteur idéal) à des fréquences beaucoup plus faibles $(\mathrm{kHz})$. La différence principale est que l'impédance d'entrée très élevée des F.E.T. qui est un facteur favorable dans ces réalisations, devient pour nous une gène car elle amène des instabilités dans le montage et une tendance aux auto-oscillations à haute fréquence.

Après de nombreux essais, notre choix s'est fixé sur les F.E.T. en arséniure de gallium GAT 4 de Plessey Electronics. Contrairement à la plupart des autres modèles, ils sont présentés en boîtiers type stripline permettant un câblage beaucoup plus compact, éliminant ainsi les instabilités rencontrées avec 
des boîtiers du type T05. D'autre part ce sont ceux qui présentent les meilleures performances au point de vue de la transconductance. La valeur typique annoncée par le constructeur est de $24 \mathrm{~mA} / \mathrm{V}$ pour $V_{\mathrm{DS}}=5 \mathrm{~V}$, $V_{\mathrm{GS}}=-1 \mathrm{~V}$. Dans les conditions de notre montage : $V_{\mathrm{Ds}}=3 \mathrm{~V}, I_{\mathrm{Ds}}=4 \mathrm{~mA}$, la valeur mesurée est de $16 \mathrm{~mA} / \mathrm{V}$. Enfin, leurs caractéristiques varient assez peu avec la température. Une modification de la tension de commande $V_{\mathrm{Gs}}$ de $10 \%$ seulement est suffisante pour retrouver à $15 \mathrm{~K}$ le même courant $I_{\mathrm{DS}}$ qu'à la température ambiante. Ceci permet de faire une grande partie des mises au point "à l'air libre ", et de n'avoir que de petits ajustements à faire pour les conditions de fonctionnement réelles.

La figure 1 donne le schéma du préamplificateur. Le montage à deux tensions permet de contrôler et de modifier de l'extérieur le point de fonctionnement des deux transistors. La contre-réaction importante en continu rend ces points de fonctionnement pratiquement indépendants du niveau d'éclairement du détecteur, celui-ci devant pouvoir être utilisé aussi bien pour la détection de fluorescences très faibles que pour la mesure des variations de transmission d'un faisceau laser relativement intense dans les mesures de double résonance.

Les caractéristiques du montage sont résumées dans le tableau I. La ligne adaptée de $68 \mathrm{ohms}$ est simplement constituée de deux fils fins maintenus parallèles; un câble de 50 ohms normal aurait amené des pertes thermiques plus importantes et diminué le gain en boucle ouverte.

\section{Performances}

En ce qui concerne le temps de réponse, le chiffre de $6 \mathrm{~ns}$ que nous donnons dans le tableau I a été obtenu de manière indirecte : la capacité parasite du cristal détecteur a été évaluée à $10 \mathrm{pF}$ par mesure de la constante de temps avec des charges plus élevées; compte tenu de l'impédance d'entrée de $600 \mathrm{ohms}$. on arrive au chiffre de $6 \mathrm{~ns}$, le temps de réponse du préamplificateur lui-même étant négligeable.

Pour contrôler directement la réponse du système, nous avons modulé le faisceau d'un laser à $\mathrm{CO}_{2}$



Fig. 1. - Schéma électrique du Préamplificateur.

F.E.T. : GAT 4(Plessey),

$R, R_{1}, R_{2}: 1,5 \mathrm{k} \Omega, R_{\mathrm{f}}: 1,2 \mathrm{k} \Omega$.

[Circuit diagram of the preamplifier.]

continu avec un dispositif électrooptique. L'association d'une lame quart d'onde, d'une cellule de Pockels et d'un analyseur permet d'obtenir, à partir d'un faisceau d'intensité $I_{0}$, une modulation autour de la valeur $I_{0} / 2$ proportionnelle à la tension appliquée sur la cellule de Pockels. La trace du bas sur la figure 2 est donc l'image de l'énergie reçue par le détecteur. L'amplitude de la modulation est de $2 \mu \mathrm{W}$ sur un fond continu de $100 \mu \mathrm{W}$. La trace du haut est la réponse du système.

Nous comparons dans le tableau II les performances de ce système du point de vue du bruit avec ce que l'on obtiendrait en utilisant le même cristal avec une charge $50 \mathrm{ohms}$. On peut constater que le rapport signal/bruit est amélioré d'un facteur voisin de 30 . Nous avons fait cette comparaison pour le système complet avec son amplificateur de sortie car celui-ci est en fait la principale source de bruit dans le cas de la charge $50 \mathrm{ohms}$.

Le bruit de photons de l'environnement à $300 \mathrm{~K}$ (colonne 1), qui est seul pris en compte dans le cas du photoconducteur idéal, a été calculé pour un détecteur

Tableau I. - Paramètres du circuit.

[Parameters of the circuit.]

Caractéristiques du montage

Gain (boucle ouverte) $: A=5$

Impédance d'entrée : $Z_{\mathrm{in}}=\frac{R_{\mathrm{F}}+\left(R_{1} / / R_{2}\right)}{1+A}=600 \Omega$

Impédance équivalente : $Z_{\text {eq }}=A \cdot Z_{\text {in }}=3 \mathrm{k} \Omega$
Contre-réaction $=\frac{R_{2}}{R_{1}+R_{2}}=1 / 2$

Impédance de sortie : $68 \Omega$

Temps de réponse : $6 \mathrm{~ns}$ 
de surface identique, ayant une longueur d'onde de coupure de $30 \mu \mathrm{m}$, et utilisé dans une bande de fréquence de $25 \mathrm{MHz}$ (temps de réponse $6 \mathrm{~ns}$ ). Le rapport signal/bruit correspondant n'est supérieur que d'un facteur 3 à celui de notre système.
Le préamplificateur refroidi représente donc une amélioration sensible par rapport au montage avec une charge 50 ohms. Les performances du détecteur ainsi mis en œuvre deviennent comparables à celles d'un photoconducteur idéal.

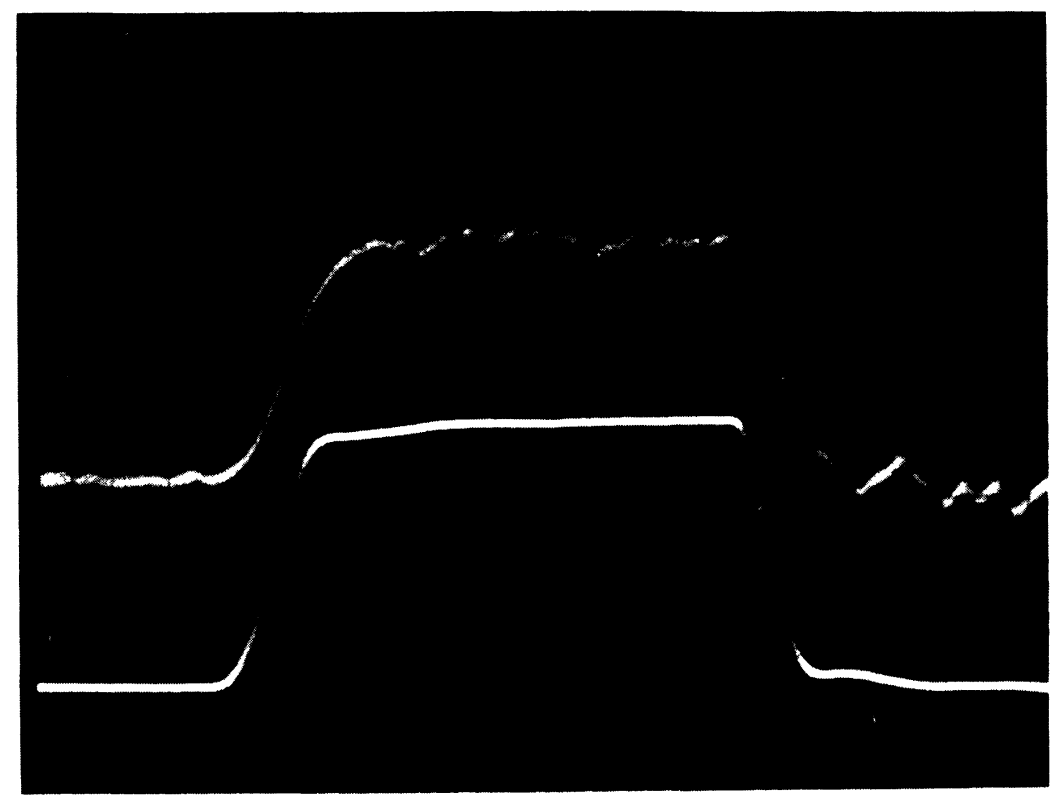

Fig. 2. - Réponse temporelle du système ( $50 \mathrm{~ns} /$ division). Trace du bas : impulsion électrique sur le cristal électrooptique; trace du haut : signal obtenu.

[Temporal response of the system (50 ns/division). Lower trace : electrical pulse on the electrooptic cristal; upper trace : signal obtained.]

Tableau II. - Performances du système.

[Performances of the system.]

\begin{tabular}{|l|c|c|c|c|c|c|c|c|}
\hline & \multicolumn{4}{|c|}{$\begin{array}{c}\text { Puissance de bruit }\left(\mu \mathrm{V}^{2}\right) \\
\text { Bande passante 25 MHz }\end{array}$} & \multicolumn{4}{c|}{ Performances $1 \mu \mathrm{W}$ de signal à $10 \mu \mathrm{m}$} \\
\hline & Environnement & Johnson & Préampli & $\begin{array}{c}\text { Ampli } \\
\text { VV 100 B }\end{array}$ & Total & $\begin{array}{c}\text { Bruit } \\
\mu \mathrm{V}\end{array}$ & $\begin{array}{c}\text { Signal } \\
\mu \mathrm{V}\end{array}$ & Signal/Bruit \\
\hline $\begin{array}{l}\text { Charge } \\
50 \Omega\end{array}$ & 0,05 & 21 & - & 450 & $471\left(^{*}\right)$ & $22\left(^{*}\right)$ & 16 & 0,73 \\
\hline $\begin{array}{l}\text { Préampli } \\
3 \mathrm{k} \Omega\end{array}$ & 240 & 84 & 1730 & 450 & $2500\left(^{*}\right)$ & $50\left(^{*}\right)$ & 960 & 20 \\
\hline $\begin{array}{l}\text { Détecteur } \\
\text { idéal }\end{array}$ & 240 & & & & & 15 & 960 & 64 \\
\hline
\end{tabular}

$\left(^{*}\right)$ Valeurs mesurées directement. 


\section{Bibliographie}

[1] Boissel, P., Gauthier-Roy, B. et Abouaf-Marguin, [5] Forrest, S. R. and Sanders, T. M. Jr., Rev. Sci. Instrum. L., J. Chem. Phys. 82 (1985) 1056. 49 (1978) 1603.

[2] Abouaf-Marguin, L., Gauthier-Roy, B. et Legay, F., [6] Hall, D. N., Aikens, R. S., Joyce, R., McCurnin, Chem. Phys. 23 (1977) 443.

T. W., Appl. Opt. 14 (1975) 450.

[3] Cryostat 9145-2HS de Santa Barbara Research Center. [7] WampaCH, H. R. and Sullivan, N. S., Rev. Sci. Instrum.

[4] Gcebel, J. H., Rev. Sci. Instrum. 48 (1977) 389. $49(1978) 1622$. 